\title{
Fatal Measles Inclusion-Body Encephalitis in Adult with Untreated AIDS, France
}

\author{
Christophe Rodriguez, Meriadeg Ar Gouilh, Nicolas Weiss, Sébastian Stroer, \\ Karima Mokhtari, Danielle Seilhean, Bertrand Mathon, Vanessa Demontant, Melissa N'Debi, \\ Guillaume Gricourt, Paul-Louis Woerther, Jean-Michel Pawlotsky, Karl Stefic, Julien Marlet, \\ Pierre-François Dequin, Antoine Guillon, Valérie Pourcher, David Boutolleau, Astrid Vabret, Sonia Burrel
}

We report a fatal case of measles inclusion-body encephalitis occurring in a woman from Romania with AIDS. After an extensive but unsuccessful diagnostic evaluation, a pan-pathogen shotgun metagenomic approach revealed a measles virus infection. We identified no mutations previously associated with neurovirulence.

$\mathrm{W}^{2}$ e report a fatal case of measles inclusion-body encephalitis in a 28-year-old woman from Romania living in France who had untreated AIDS. She was initially admitted to the hospital on September 22, 2018 (day 0), for afebrile generalized motor seizure that began focally in the right lower limb. Results of magnetic resonance imaging (MRI) were initially unremarkable, but electroencephalogram (EEG) results showed slight abnormalities related to slow frontal activity. The patient recovered fully and was discharged with antiepileptic therapy. Of note, the patient had stopped antiretroviral therapy (ART) 1 year earlier and declined to restart therapy after this hospital admission.

Author affiliations: Henri Mondor Hospital, Assistance Publique des Hôpitaux de Paris, University of Paris-Est, Créteil, France (C. Rodriguez, V. Demontant, M. N'Debi, G. Gricourt, P.-L. Woerther, J.-M. Pawlotsky); National Reference Laboratory for Measles, Mumps, and Rubella, University Hospital of Caen, Normandie Université, Caen, France (M. Ar Gouilh, A. Vabret); Pitié-Salpêtrière Hospital, Assistance Publique des Hôpitaux de Paris, Sorbonne-Université, Paris, France (N. Weiss, S. Stroer, K. Mokhtari, D. Seilhean, B. Mathon, V. Pourcher, D. Boutolleau, S. Burrel); National Reference Center for Herpesviruses, Paris (D. Boutolleau, S. Burrel); University Hospital of Tours, University of Tours, Tours, France (K. Stefic, J. Marlet, P.-F. Dequin,

A. Guillon); National Reference Center for HIV, Tours (K. Stefic, J. Marlet)

DOI: https://doi.org/10.3201/eid2609.200366
In the next week, she had several relapses of focal seizures, requiring hospital readmission on day 7 . Despite antiepileptic therapy adjustments, the myoclonic seizures persisted and became resistant to high doses of anticonvulsants and clonazepam add-on therapy (day 31). Consequently, the patient was hospitalized in intensive care unit. EEG results showed a pattern of frontal-lobe epilepsy. MRI results showed hyperintense cortical signals in frontal and left temporal cortex without hemorrhage lesions and without any signs of cerebral venous thrombosis. Biologic investigation revealed HIV replication and $26 / \mathrm{mm}^{3}$ CD4 T-cell count at day 35. We initiated antiretroviral medications on day 43 .

During her hospitalization, the patient showed a gradual impairment of consciousness (Glasgow coma score 6 on day 61) and was mechanically ventilated. MRI results showed increase of the cortical hyperintensities, and EEG results showed diffuse encephalopathy pattern (Figure 1). We analyzed cerebrospinal fluid (CSF) samples taken on days 37, 62, and 64 for pathogens: viruses (herpes simplex virus, varicella zoster virus, enterovirus, cytomegalovirus, Epstein-Barr virus, human herpesvirus 6, HIV, and polyomavirus JC), bacterial and mycobacteria, fungi (Aspergillus spp., Cryptococcus neoformans), and parasites (Toxoplasma gondii); no pathogens were detected. All CSF were paucicellular with protein and glucose levels within reference levels. Results of testing for autoimmune antibodies were also negative.

We performed a brain biopsy of the left frontal lobe on day 71 to determine the cause of encephalitis by the underlying neurologic symptoms, abnormal imaging features, and biologic findings. Neuropathology analysis revealed scarce inflammatory activation of glial cells (Figure 2). Because all the first-line microbiology testing assays remained negative on the 
biopsy, we considered using shotgun metagenomic (SMg) for panpathogen RNA/DNA detection to analyze the clinical samples with an unbiased approach. In brief, we performed an extraction combining bead beating and chemical and enzymatic lysis before library preparation. We performed sequencing on NextSeq500 with High Output Kit version 2.5 (300 cycles) (Illumina, https://www.illumina.com) (1). We analyzed sequencing data using MetaMIC software, which performed microorganism identification, genome reconstruction, and variant calling (1). A total of 5 samples were tested by SMg: CSF (day 61), bronchoalveolar lavage (days 63 and 76), brain biopsy (day 71), and whole blood (day 68). Only the brain
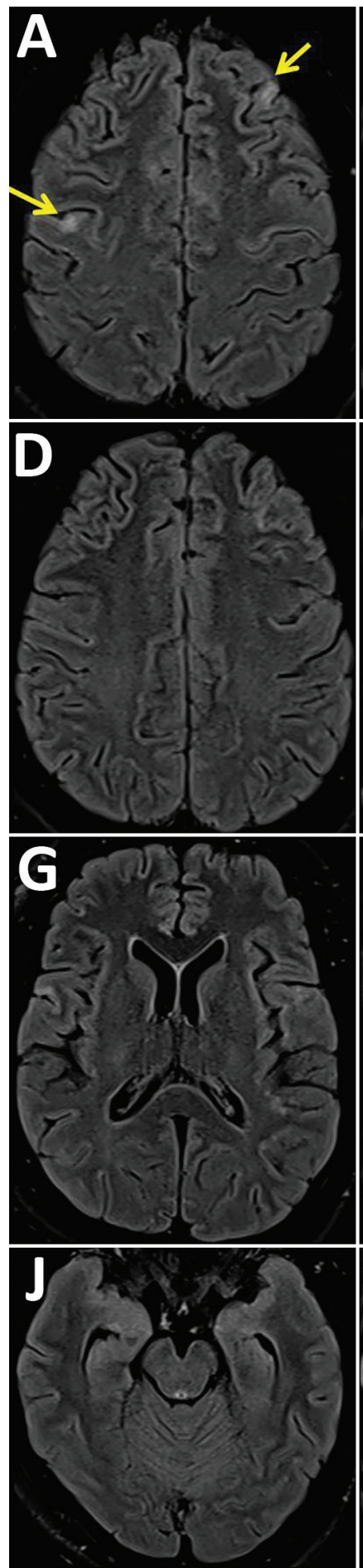
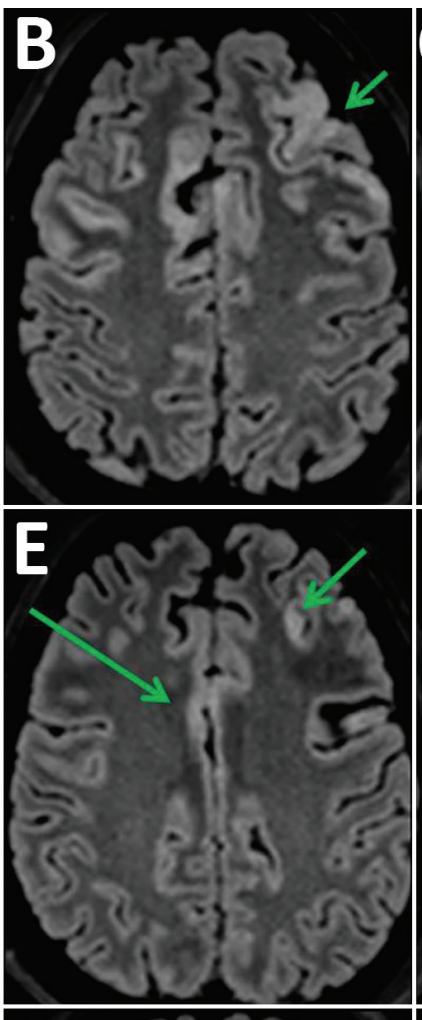

\section{H}
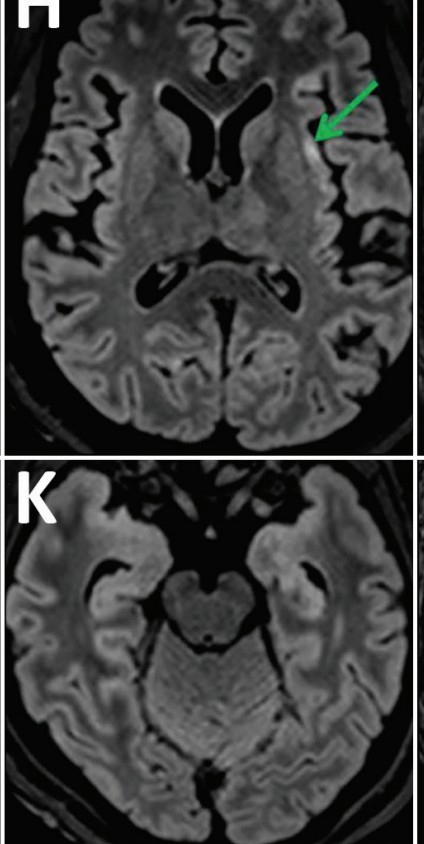
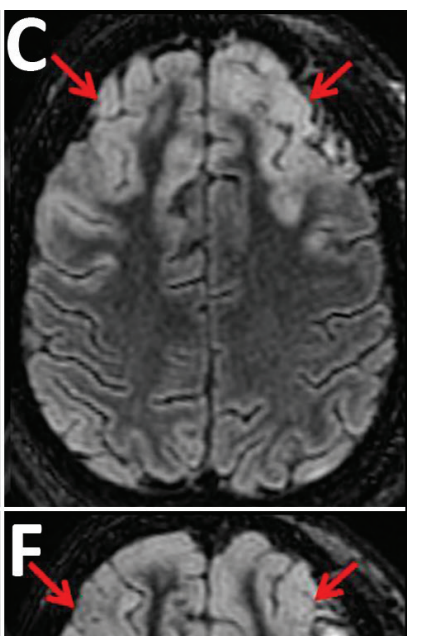

Figure 1. Fluid-attenuated inversion recovery images of magnetic resonance examinations of the brain in a 28-year-old woman from Romania with untreated AIDS and measles inclusion-body encephalitis. Images were taken 1 week (A, D, G, J), 2 weeks $(B, E, H, K)$, and 5 weeks (C, $\mathrm{F}, \mathrm{I}, \mathrm{L})$ after hospital admission, at the same brain levels. The first examination shows focal cortical hyperintensities (yellow arrows) in the left and right frontal cortex. After 2 weeks, these cortical hyperintensities have widened and are spreading to the cingulum and the insula (green arrows). At 5 weeks, cortical hyperintensities involve a larger part of the neocortex, but also spread to the basal ganglia, amygdala (red arrows), and hippocampus (the hippocampus changes may also be induced by status epilepticus) and to the posterior areas of the pons. 
Figure 2. Histology and immunohistochemical staining of the cerebral cortex in a 28-year-old woman from Romania with untreated AIDS and measles inclusion-body encephalitis. A) Histology shows moderate increased cellular density and absence of nuclear inclusion bodies. Hematoxylin and eosin stain; original magnification $\times 100$. B) Immunohistochemical staining microglial activation with high CD163 immunoreactivity Anti-human CD163 monoclonal antibody; original magnification $\times 100$ and (inset) $\times 400$.

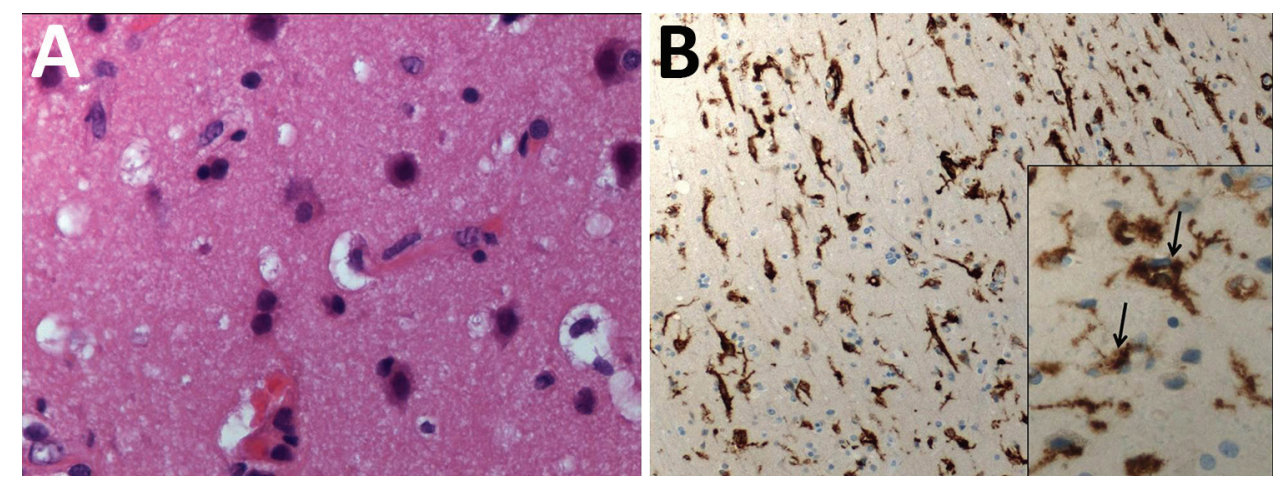

biopsy sample was found to be positive for measles virus $(\mathrm{MeV}) ;>4,800,000$ of $2 \times 150$ paired reads were assigned to the virus. These results were confirmed by specific $\mathrm{MeV}$ real-time reverse transcription PCR.

The detected $\mathrm{MeV}$ sequences in the brain biopsy enabled the reconstruction of a nearly-complete genome assembly $(99.5 \%$ with a median depth coverage >25,000; GenBank accession no. MN893225). BLAST analysis (https://blast.ncbi.nlm.nih.gov/ Blast.cgi) showed this $\mathrm{MeV}$ exhibited 99.3\% identity $(15,762 / 15,876 \mathrm{nt})$ with the closest available fully sequenced B3 genotype (MVs/California.USA/05.14/ [B3]; GenBank accession no. KY969477). These results excluded infection by a $\mathrm{MeV}$ vaccine strain and oriented toward a European lineage origin. However, it was not possible to determine the precise origin of the virus because a high number of $\mathrm{MeV}$ harboring an identical C-terminal hypervariable domain (450 nt) of the nucleoprotein N gene N-450, the only genetic data available on the World Health Organization Measles Nucleotide Surveillance database (http:/ / www.whomeasles.org) (2), were co-circulating in Europe at the time (data not shown) (3-6). In addition, we identified no previously reported mutations suspected for neurovirulence $(7,8)$.

We observed a mutational hotspot within the virus-encoded matrix protein (M). Of interest, the F1 5\% end of the fusion protein $(F)$ contained the most variable sites found along the genome. This hydrophobic F1 part is associated with hyperfusogenicity and neurodegenerative disorders (data not shown) $(7,8)$.

Several characteristics supported a diagnosis of measles inclusion-body encephalitis (MIBE): an immunocompromised patient, undetectable MeV RNA with no intrathecal synthesis of $\mathrm{MeV}$ antibodies in CSF (MeV-specific IgG were detected in serum), and scarce inflammatory infiltrates on brain biopsy despite the absence of characteristic inclusions or multinucleated giant cells (9). Moreover, the retrospective clinical investigation revealed that the patient, who was not vaccinated against measles, had close contact with a sibling who was acutely ill with measles in April 2018 in Romania. Because of late MIBE diagnosis and despite supportive treatment, the patient's neurologic status continued to deteriorate rapidly, and she died at day 109 with severe brain damage exemplified by pejorative MRI evolution (Figure 1), showing bilateral, symmetric, and diffuse distribution of lesions during days 59-94.

Ongoing measles resurgence may lead to an increase of measles-induced encephalitis cases with life-threatening outcomes. We report here a fatal case in a woman with AIDS who had an encephalitic syndrome with no initial clear etiologic diagnosis, retrospectively tagged as MIBE. The patient did not receive ribavirin therapy (10) for $\mathrm{MeV}$ infection because the first-line extensive diagnostic testing was unsuccessful. As a last resort, a SMg approach detected $\mathrm{MeV}$ in a brain biopsy, despite the known result that CSF $\mathrm{MeV}$ detection in MIBE is often negative (9-11). Of interest, the brain biopsy did not reveal histopathologic features consistent with MIBE; we observed no immunoreactive inclusions or multinucleated giant cells within glial cells or neurons $(10,11)$. However, MIBE lesions are scanty and can be missed in a small biopsy sample. Unfortunately, there was no material available for electron microscopy, and an autopsy was not done.

The encephalitic syndrome developed in this unvaccinated patient $\approx 6$ months after a close contact with a documented measles case-patient; however, she did not report any rash or clinical symptoms of measles infection. It is noteworthy that $\mathrm{MeV}$ real-time reverse transcription PCR performed on the brain biopsy sample could have been sufficient to detect the virus. However, in this case, the advantage of SMg for the diagnosis of encephalitis is that, aside from pathogen identification, it was possible to generate 
full genome sequence for B3-genotype MeV. In conclusion, this case highlights the advantage to have a reliable pan-pathogen $\mathrm{SMg}$ tool to diagnose atypical encephalitis with no clear etiology on an early brain biopsy sampling.

\section{Acknowledgments}

We thank the World Health Organization Measles Nucleotide Surveillance database administrator, curators, and national reference labs that contribute to this global surveillance system, especially Kevin Brown, Luiza Ustea, Mihaela Lazar, Jeff Connel, and Fabio Magurano.

\section{About the Author}

Dr. Rodriguez is an assistant professor in the Department of Microbiology at Henri Mondor Hospital, University of Paris-Est-Créteil, Créteil, France, and the head of the institution's genomics platform. He has implemented a clinical metagenomics technique in diagnostic routine and is working on the exploration of complex infectious diseases using this tool.

\section{References}

1. Rodriguez C, Jary A, Hua C, Woerther P-L, Bosc R, Desroches M, et al.; Multidisciplinary Necrotizing Fasciitis Study Group. Pathogen identification by shotgun metagenomics of patients with necrotizing soft-tissue infections. Br J Dermatol. 2019; https:/ / doi.org/10.1111/ bjd.18611

2. Rota PA, Brown K, Mankertz A, Santibanez S, Shulga S, Muller CP, et al. Global distribution of measles genotypes and measles molecular epidemiology. J Infect Dis.
2011;204(Suppl 1):S514-23. https:/ / doi.org/10.1093/infdis/ jir118

3. Katoh K, Standley DM. MAFFT multiple sequence alignment software version 7: improvements in performance and usability. Mol Biol Evol. 2013;30:772-80. https:/ / doi.org/ 10.1093/molbev/mst010

4. Drummond AJ, Rambaut A. BEAST: Bayesian evolutionary analysis by sampling trees. BMC Evol Biol. 2007;7:214. https:// doi.org/10.1186/1471-2148-7-214

5. Drummond AJ, Ho SYW, Phillips MJ, Rambaut A. Relaxed phylogenetics and dating with confidence. PLoS Biol. 2006;4:e88. https://doi.org/10.1371/journal.pbio.0040088

6. Darriba D, Taboada GL, Doallo R, Posada D. jModelTest 2: more models, new heuristics and parallel computing. Nat Methods. 2012;9:772. https://doi.org/10.1038/nmeth.2109

7. Hashiguchi T, Fukuda Y, Matsuoka R, Kuroda D, Kubota M, Shirogane Y, et al. Structures of the prefusion form of measles virus fusion protein in complex with inhibitors. Proc Natl Acad Sci USA. 2018;115:2496-501. https://doi.org/10.1073/pnas.1718957115

8. Plattet P, Alves L, Herren M, Aguilar HC. Measles virus fusion protein: structure, function and inhibition. Viruses. 2016;8:112. https:/ / doi.org/10.3390/v8040112

9. Fisher DL, Defres S, Solomon T. Measles-induced encephalitis. QJM. 2015;108:177-82. https://doi.org/10.1093/qjmed/hcu113

10. Baldolli A, Dargère $S$, Cardineau $E$, Vabret $A$, Dina J, de La Blanchardière A, et al. Measles inclusion-body encephalitis (MIBE) in a immunocompromised patient. J Clin Virol. 2016;81:43-6. https:/ / doi.org/10.1016/j.jcv.2016.05.016

11. Bitnun A, Shannon P, Durward A, Rota PA, Bellini WJ, Graham C, et al. Measles inclusion-body encephalitis caused by the vaccine strain of measles virus. Clin Infect Dis. 1999;29:855-61. https:/ / doi.org/10.1086/520449

Address for correspondence: Dr. Sonia Burrel, Assistance Publique-Hopitaux de Paris, La Pitie Salpetriere, University Hospital, Virology Department, 83 boulevard de l'hopital Paris, Île-de-France 75013, France; email: sonia.burrel@aphp.fr 